\title{
Bowel Obstruction Following Lap Appendectomy and Open Appendectomy
}

Osama Abdullah Abdul Raheem and Ehab Motawa El Hussieny

Department of General Surgery, Faculty of Medicine, Aswan University

*Corresponding Author: Osama Abdullah Abdul Raheem, Phone No.: (+20)01155520802,

E-mail: Osamaaloraby@yahoo.com

\begin{abstract}
Background: acute appendicitis is a common indication for abdominal surgery with a life-time incidence between 7 and 9\% and appendectomy is one of the most common surgical procedures. Open appendectomy (OA) performed through the right lower quadrant incision was first described in 1894. It has become the standard treatment of choice for acute appendicitis, due to its efficacy and safety.

Objective: the aim of the present study was to compare the frequency of readmissions due to bowel obstruction (BO) after open versus laparoscopic surgery performed for suspected acute appendicitis.

Patients and Methods: this was performed in Aswan University hospital, on 100 patients from April 2015 to April 2017 who underwent laparoscopic and open appendectomy. The merits and drawbacks of LA and OA were explained to all of the patients with diagnosis of acute appendicitis.

Results: a statistically significant difference was found when comparing the operative time of the LA and OA. The operating times in OA and LA were 16-37 min (mean 24.7) and 19-45 min (mean 33.1), respectively. The OA was shorter in duration, with a $\mathrm{P}$ value less than or equal to 0.05 (Mann-Whitney test).

Conclusion: laparoscopic appendectomy is equally safe, and can provide less postoperative morbidity in experienced hands, as open appendectomy. Most cases of acute appendicitis can be treated laparoscopically. Laparoscopic appendectomy is a useful method for reducing hospital stay, complications and return to normal activity.
\end{abstract}

Keywords: Bowel obstruction, Appendectomy, Open appendectomy

\section{INTRODUCTION}

Successful appendectomy was first described by McBurney ${ }^{(\mathbf{1})}$. The open surgical approach remained the gold standard for nearly a century. The lifetime risk of developing appendicitis is between 7 and $9 \%$ with evidence of increasing incidence ${ }^{(2,3)}$.

With the advance of minimal invasive surgery, new approaches for the existing operations have been proposed. Semm ${ }^{(4)}$ first described the laparoscopic approach for acute appendicitis in 1983. Now laparoscopic appendicectomy (LA) has become common and an acceptable approach in the management of acute appendicitis ${ }^{(5)}$. LA is associated with decreased postoperative pain, more rapid return to daily activities, and improved cosmetic results ${ }^{(6)}$. However, the literature has shown the association of laparoscopy with specific adverse events such as increased intra-abdominal abscess and hospital costs ${ }^{\left({ }^{(6)}\right.}$.

Despite OA being associated with low morbidity and mortality rates the popular minimally invasive approach showed more advantages such as less wound infection, less pain, and faster recovery in the cost of more operating time and hospital $\cos ^{(7,8)}$. LA can have extra benefits for female patients as decreasing adhesions and fertility problems and better cosmetic ${ }^{(9)}$.

Adhesion formation is now one of the most common causes of intestinal obstruction. Late complications due to intra-abdominal adhesions include chronic abdominal pain, small bowel obstruction (SBO), and female infertility. These chronic conditions can result in a major impairment for the patient, a challenge to treat, and represent a major cost for society ${ }^{\mathbf{1 0 . 1 1})}$. Reduced adhesion formation is a substantive long term advantage of laparoscopic
appendectomy.The adhesion rateafter open appendectomy more than $80 \%$ compared to $10 \%$ after laparoscopic appendectomy, when patients were laparoscoped three months after surgery ${ }^{\mathbf{( 1 0 )}}$. It has been shown that the tissue trauma of the incision increases the total inflammatory response, thereby inhibiting fibrinolysis and promoting fibroblast migration and collagen formation.

\section{AIM OF THE WORK}

The aim of the present study is to compare the frequency of readmissions due to bowel obstruction (BO) after open versus laparoscopic surgery performed for suspected acute appendicitis.

\section{PATIENTS AND METHODS}

This was performed in Aswan University hospital, on 100 patients from April 2015 to April 2017 who underwent laparoscopic and open appendectomy. The merits and drawbacks of LA and OA were explained to all of the patients with diagnosis of acute appendicitis. The study was approved by the Ethics Board of Aswan University and an informed written consent was taken from each participant in the study.

The diagnosis of acute appendicitis was mainly clinical. Several methods have been suggested to diminish the diagnostic error like U/S and CT abdomen if diagnosis is not clear. The symptoms of appendicitis can initially be difficult to differentiate from gastroenteritis. Early symptoms may include vague bloating, indigestion and mild pain which generally is perceived as being in the area of the umbilicus. As the infection worsens, the pain becomes more prominent in 
the right lower quadrant. There is usually nausea, vomiting and loss of appetite.

Many other conditions can mimic appendicitis such as gastroenteritis, kidney stones, urinary infections, ulcerative colitis and Crohn's disease. In women, problems such as ovarian cysts and pelvic infections can mimic appendicitis. The most accurate non-invasive method of diagnosis is ultrasonography but this is not totally reliable. The history and physical examination will generally lead to the correct diagnosis.

Our exclusion criteria for this study were being patients showing signs of generalized peritonitis, having a palpable mass in RLQ suggesting appendicular mass or abscess, or being pregnant or appendectomy was performed as a part in a more extensive surgical procedure colorectal resection or when the operation was extended because of an advanced appendicitis (e.g., ileocecal resection). Also, the patient was excluded when the appendectomy was accompanied with another concurrent intra-abdominal surgical procedure (e.g., oophorectomy, Meckel diverticulum) and patients with carcinoma of the appendix.

The patient classified blindly into two groups, group A for LA and group B for OA. Patients who gave their informed consent were randomized to either LA or OA groups. The randomization technique was by having patient open a concealed envelope from a randomized order of envelops by a blinded technician.

All of the patients received prophylactic efteriaxone (1gram, every 12hours) and metronidazole (500mg, every 8hours). All of the surgeries were performed by the same surgeon.

\section{Laparoscopic appendectomy techniques:}

After induction of general anesthesia, patients were placed in the supine position and a Foley catheter was placed to decompress the bladder. All patients received preoperative intravenous antibiotics.

Pneumoperitoneum was induced with carbon dioxide up to a pressure of $12-14 \mathrm{mmHg}$ using a veress needle. Supraumbilical incision done A 10- -mm-port was then placed $30^{\circ}$ laparoscope was used for the procedureand the peritoneal cavity was accessed. Two additional $5-\mathrm{mm}$ ports were placed in the left lower quadrant and in the midline suprapubic position, ensuring identification and avoidance of the dome of the bladder. Instruments of 5-mm were used for the procedure. Once the appendix was localized, dissected free, and grasped, the mesoappendix was taken down under tension using hook electrocautery. Once the base was cleared, it was ligated, and the appendix was resected using 5 -mm polydioxanoneendoloops at the base and the appendix was sharply excised. The appendix was then removed through the umbilical port itself. The appendix was placed in an endocatch bag before removal.

After removal of the appendix, the abdomen and pelvis were irrigated thoroughly with warm sterile saline and then the wound was closed.

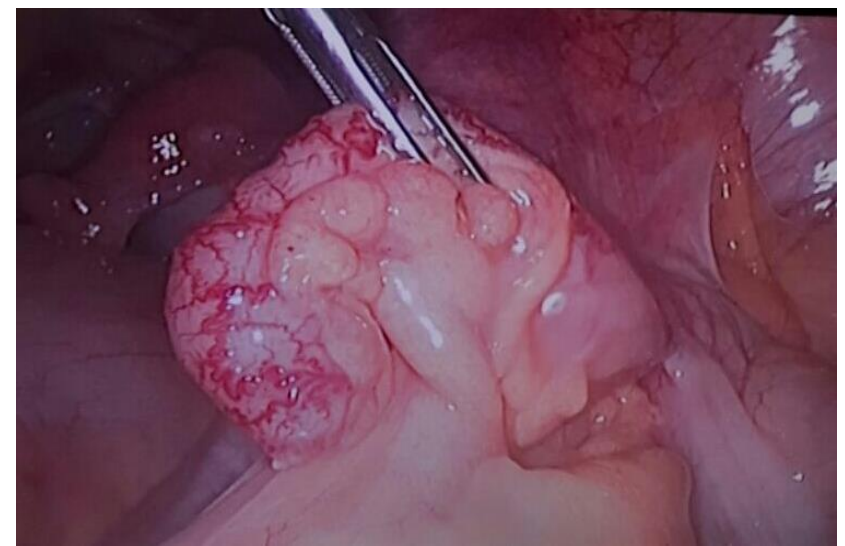

Figure (1): Showed inflamed appendix.

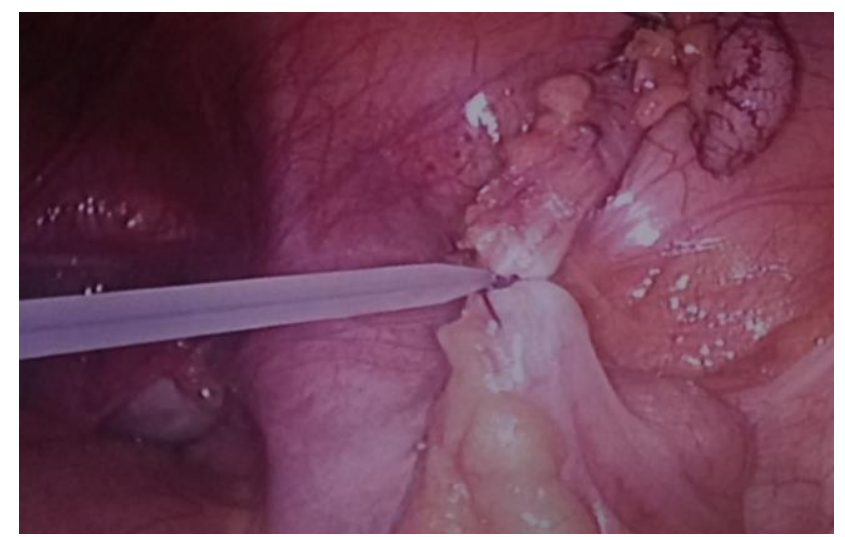

Figure (2): Showed ligation of base with endoloop.

After the operation all of the patients were NPO and received antibiotics for 48hours. The routine analgesic used for patients was morphine $(5 \mathrm{mg}$ intramuscular, every 8hours). Soft diet was started after 24 hours and patients were discharged after normal diet was tolerated. Post operation follow up visits were in weeks 1,2 and 4 . Patients were asked to contact the therapy team in case of any abdominal problem.

\section{Open Appendectomy}

The classic transverse incision can be made with two thirds of the incision lateral to McBurney's point.

A scalpel is used to incise the epidermis and the dermis. Bovieelectrocautery is used to dissect down to the external oblique aponeurosis. The aponeurosis is opened in a superolateral to inferomedial direction along the direction of its fibers to expose the internal oblique muscle. The internal oblique muscle is bluntly divided perpendicular to the direction of its fibers. The transverse abdominal muscle is similarly divided and the peritoneum is identified. The peritoneum is grasped with forceps and incised with a 15-blade knife. Attention is now focused on locating the appendix. If the cecum is visualized, it can be used as a guide to help identify the appendix. Babcock forceps can be used to grasp the taeniae coli and advanced until the appendix is externalized.Alternatively, a finger can be swept around the cecum, beginning superolaterally and 
continuing inferomedially to locate the appendix. Once identified, the mesoappendix is dissected and the appendiceal vessels are divided between clamps and ligated with silk sutures.

The stump is then buried and the suture tied.It is not necessary to bury the appendix stump especially if the caecum is inflamed.

\section{Closure and Drainage:}

The incision closed inlayers with absorbable sutures - the skin, in uncomplicated cases, may be closed with a subcuticular suture. If pus is encountered, the skin may be left open or loosely approximated. Drains may be used when an abscess is encountered.

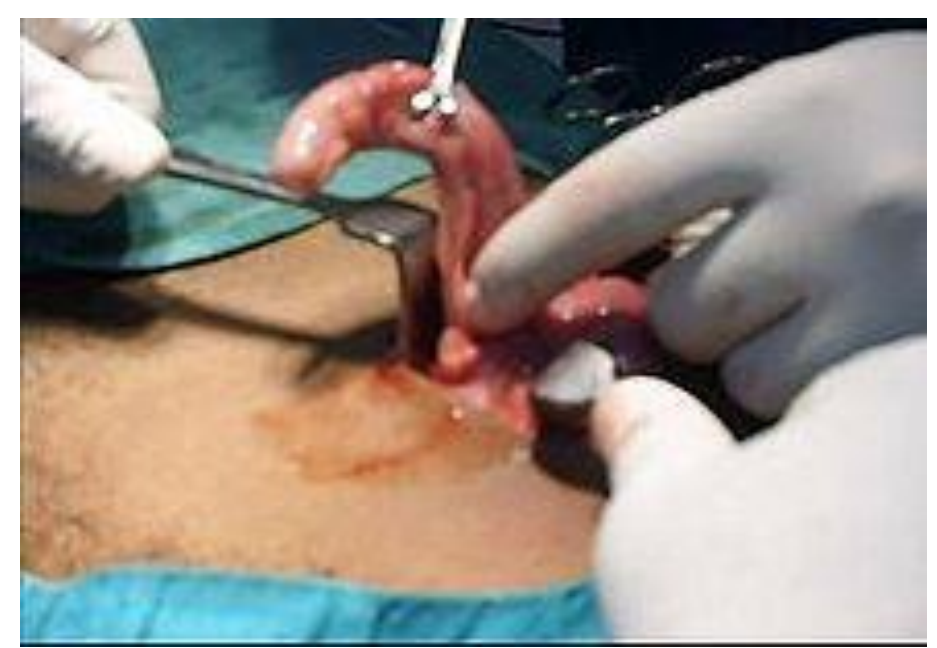

Figure (3): Open appendectomy

\section{RESULTS}

The total number of 100 patients, in two groups of open and laparoscopic appendectomy, was followed up for six month in this study. The LA group was 50 patient and OA group also 50. The mean age was 18.3 for group A and 19 for group B (fig. 4). The group A was 30 male and 20 female and group B was 35 male and 15 female (table. 1).

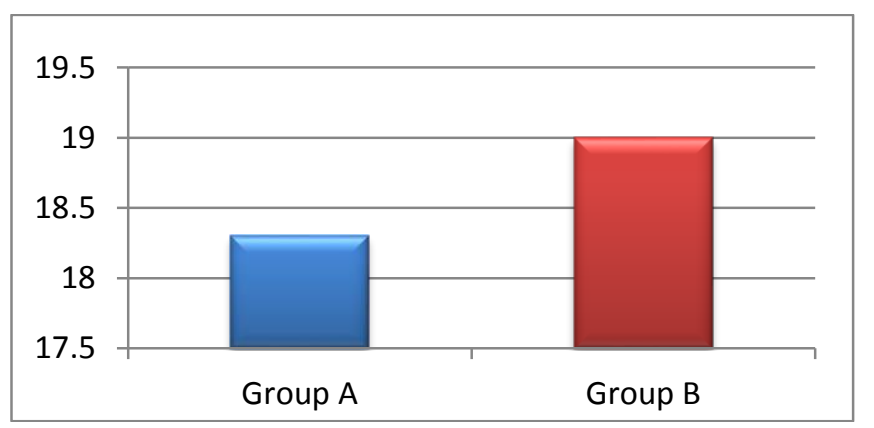

Figure (4): Mean age in two groups.

Table (1): Male and female ratio in both groups.

\begin{tabular}{|l|c|c|c|c|}
\hline & Male & $\%$ & Female & $\%$ \\
\hline Group A & 30 patients & $60 \%$ & 20 patients & $40 \%$ \\
\hline Group B & 35 patients & $70 \%$ & 15 patients & $30 \%$ \\
\hline
\end{tabular}

No intraoperative complication was encountered in operations. There was no conversion to open surgery in LA operations.

No complication for group A during the period of follow up exceptchronic pain at the port site and improved with time and analgesia.

Group B about 10 patient readmitted with abdominal pain and distension, $\mathrm{x}$ rays abdomen done for them and showed intestinal obstructionand diagnosed as adhesive intestinal obstruction 8 of them treated conservative fluid support, NGT and enema and discharged after improvement, the other two patient not improved with medication and treated by open surgery and we found adhesion at the site of wound adhesolysis done for them and improved after one week and discharged.

Table (2): Post-operative follow up for development of intestinal obstruction for two groups

\begin{tabular}{|l|c|c|}
\hline & $\begin{array}{c}\text { Group } \\
\text { A }\end{array}$ & Group B \\
\hline $\begin{array}{l}\text { Symptom of intestinal } \\
\text { obstruction abdominal } \\
\text { pain ,distention } \\
\text {,vomiting and } \\
\text { absolute constipation }\end{array}$ & No & $\begin{array}{c}\text { 10 Patients } \\
\text { 8 Patient treated } \\
\text { conservative } \\
\text { 2 patients treated } \\
\text { with surgical } \\
\text { intervention }\end{array}$ \\
\hline
\end{tabular}

\section{Intraoperative time:}

A statistically significant difference was found when comparing the operative time of the LA and OA. The operating times in OA and LA were 16-37 min (mean 24.7) and 19-45 min (mean 33.1), respectively. The OA was shorter in duration, with a $\mathrm{P}$ value less than or equal to 0.05 (Mann-Whitney test).

\section{Postoperative pain:}

Using the Wong-Baker FACES Pain Rating Scale, it was found that $16 \%$ of the patients in the LA group had pain whereas $32 \%$ of the patients in the OA group had pain.

\section{Length of the hospital stay:}

The hospital stay decreased significantly with LA. The mean postoperative hospital stay in the LA group was 1.8 days, whereas in the OA group, it was 2.4 days $(\mathrm{P} \leq 0.05)$ (Mann-Whitney test).

\section{Analgesic requirement:}

Postoperative pain was evaluated by the number of analgesic injections required. Patients who underwent LA and OA were treated with intravenous or intramuscular Ketolac injections at 12-h intervals. Analgesic requirements decrease significantly with laparoscopy.

\section{Wound infection:}

Wound infection or port-site infection was recorded in four patients in the OA group, and treated with daily dressing and antibiotic 


\section{DISCUSSION}

Postoperative adhesions are quite common, especially not only after surgery of the lower part of the abdomen such as gynecological and colorectal surgery but also after appendectomy. Late complications due to intra-abdominal adhesions include chronic abdominal pain, bowel obstruction (BO), and female infertility. These chronic conditions can result in a major impairment for the patient, a challenge to treat, and represent a major cost for society. In Sweden, costs related to admissions due to abdominal adhesions are estimated to about $€ 40$ million to $€ 60$ million per year ${ }^{(11)}$.Abdominal adhesions are the most common cause of BO requiring surgical intervention. The lifetime risk of postoperative bowel obstruction after abdominal surgery with a frequency of up to $25 \%$ after some procedures has been reported. The incidence of bowel obstruction after appendectomy has historically been reported to be between $0.7 \%$ and $10.7 \%{ }^{(12.13)}$.

Type and grade of surgical trauma seem to play an important role in adhesion formation. There are documented advantages of laparoscopic procedures in general, as compared with open surgery, in reducing postoperative adhesion formation by minimizing the trauma to the peritoneal surface ${ }^{(\mathbf{1 4})}$.The laparoscopic surgical techniques lead to fewer intra-abdominal adhesions by reducing tissue trauma, which in turn reduces circulating inflammatory mediators ${ }^{(\mathbf{1 5})}$.

Open surgery seems to increase the risk of BO at least 4-fold compared with laparoscopic surgery for most of the abdominal surgical procedures ${ }^{(16)}$. The laparoscopic approach appears to decrease the risk of adhesion formation by $45 \%$ as well as decreasing the need for adhesion-related reintervention to $0.8 \%$ after appendectomy ${ }^{(\mathbf{1 7})}$. A recent study by Isaksson et al..$^{(\mathbf{1 8})}$ found a significant difference in the rate of postoperative BO: $1.0 \%$ in the open group, and $0.4 \%$ in the laparoscopic group. In our study we confirm low intestinalcomplication following lap app so become trend in our hospital to do lap app in cases of acute appendicitis.

\section{CONCLUSION}

Laparoscopic appendectomy is equally safe, and can provide less postoperative morbidity in experienced hands, as open appendectomy. Most cases of acute appendicitis can be treated laparoscopically. Laparoscopic appendectomy is a useful method for reducing hospital stay, complications and return to normal activity.

\section{REFERENCES}

1. McBurney $\mathbf{C}$ (1894): The incision made in the abdominal wall in cases of appendicitis, with a description of a new method of operating. Annals of Surgery, 20(1): 38-43.

2. Addiss DG, Shaffer N, Fowler BS et al. (1990): The epidemiology of appendicitis and appendectomy in the
United States. American Journal of Epidemiology, 132(5): 910-925.

3. Buckius MT, McGrath B, Monk J et al. (2012): Changing epidemiology of acute appendicitis in the United States: study period 1993-2008. Journal of Surgical Research, 175(2): 185-190.

4. Semm K (1983): Endoscopic appendectomy. Endoscopy, 15(2): 59-64.

5. Litynski GS (1998): Kurt Semmand the fight against skepticism: endoscopic hemostasis, laparoscopic appendectomy, and Semm's impact on the laparoscopic revolution. Journal of the Society of Laparoendoscopic Surgeons, 2(3): 309-313.

6. Sauerland S, Lefering R, and Neugebauer EA (2004): Laparoscopic versus open surgery for suspected appendicitis.

https://www.researchgate.net/.../47358480_Laparoscopic _versus_open_surgery_for_sus

7. Shaikh AR, Sangrasi AK and Shaikh GA (2009): Clinical outcomes of laparoscopic versus open appendectomy. JSLS., 13(4):574-80.

8. Heikkinen TJ, Haukipuro $K$ and Hulkko A (1998): Cost-effective appendectomy. Open or laparoscopic? A prospective randomized study. Surg Endosc., 12(10):1204-8.

9. Tzovaras G, Liakou P, Baloyiannis I et al. (2007): Laparoscopic appendectomy: differences between male and female patients with suspected acute appendicitis. World J Surg., 31(2):409-13.

10. Cuschieri A (1997): Appendectomy-laparoscopic or open? Surg Endosc., 11: 319-320.

11. Tingstedt B, Isaksson J and Andersson R (2007): Longterm follow-up and cost analysis following surgery for small bowel obstruction caused by intra-abdominal adhesions. Br J Surg., 94:743-748.

12. Tsao KJ, St Peter SD, Valusek PA et al. (2007): Adhesive small bowel obstruction after appendectomy in children: comparison between the laparoscopic and open approach. J Pediatr Surg., 42:939-942.

13. Zbar RI, Crede WB, McKhann CF et al. (1993): The postoperative incidence of small bowel obstruction following standard, open appendectomy and cholecystectomy: a six-year retrospective cohort study at Yale-New Haven Hospital. Conn Med., 57:123-127.

14. Gutt CN, Oniu T, Schemmer $P$ et al. (2004): Fewer adhesions induced by laparoscopic surgery? Surg Endosc., 18:898-906.

15. Garrard CL, Clements RH, Nanney L et al. (1999): Adhesion formation is reduced after laparoscopic surgery. Surg Endosc., 13: 10-13.

16. Angenete E, Jacobsson A, Gellerstedt $M$ et al. (2012): Effect of laparoscopy on the risk of small-bowel obstruction: a population-based register study. Arch Surg., 147:359-365.

17. Ouaïssi M, Gaujoux $S$, Veyrie $N$ et al. (2012): Postoperative adhesions after digestive surgery: their incidence and prevention: review of the literature. J Visc Surg., 149:104-114.

18. Isaksson K, Montgomery A, Moberg AC et al. (2014): Long-term follow-up for adhesive small bowel obstruction after open versus laparoscopic surgery for suspected appendicitis. Ann Surg., 259:1173-1177. 